\title{
Diet, Lifestyle, Smoking
}

\section{Lale Tokgozoglu, Vedat Hekimsoy, Giuseppina Costabile, Ilaria Calabrese, and Gabriele Riccardi}

\section{Contents}

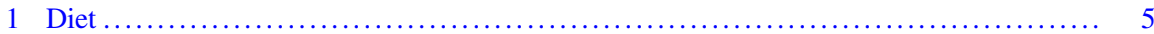

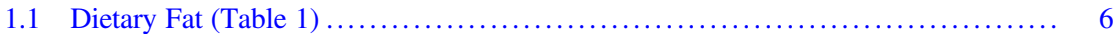

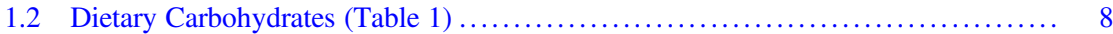

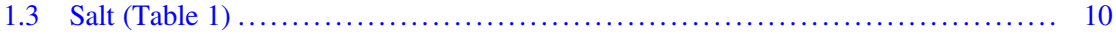

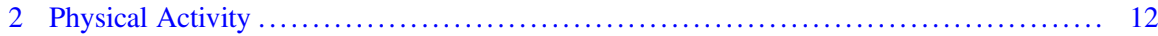

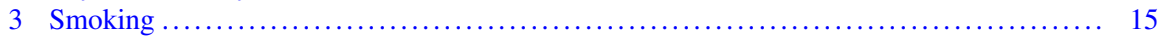

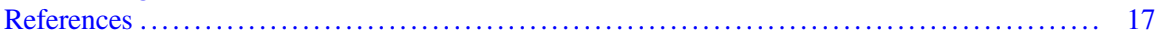

\section{Abstract}

Cardiovascular disease is the leading cause of death globally The past few decades have shown that especially low- and middle-income countries have undergone rapid industrialization, urbanization, economic development and market globalization. Although these developments led to many positive changes in health outcomes and increased life expectancies, they all also caused inappropriate dietary patterns, physical inactivity and obesity. Evidence shows that a large proportion of the cardiovascular disease burden can be explained by behavioural factors such as low physical activity, unhealthy diet and smoking. Controlling these risk factors from early ages is important for maintaining cardiovascular health. Even in patients with genetic susceptibility to cardiovascular disease, risk factor modification is beneficial.

Despite the tremendous advances in the medical treatment of cardiovascular risk factors to reduce overall cardiovascular risk, the modern lifestyle which has led to

\footnotetext{
L. Tokgozoglu $(\bowtie) \cdot$ V. Hekimsoy

Department of Cardiology, Hacettepe University Faculty of Medicine, Ankara, Turkey

G. Costabile · I. Calabrese · G. Riccardi

Department of Clinical Medicine and Surgery, Federico II University, Naples, Italy

e-mail: riccardi@unina.it
} 
greater sedentary time, lower participation in active transport and time spent in leisure or purposeful physical activity, unhealthy diets and increased exposure to stress, noise and pollution have diminished the beneficial effects of contemporary medical cardiovascular prevention strategies. Therefore attenuating or eliminating these health risk behaviours and risk factors is imperative in the prevention of cardiovascular diseases.

\section{Keywords}

Cardiovascular diseases $\cdot$ Diet $\cdot$ Lifestyle $\cdot$ Prevention

The global burden of disease has dramatically shifted from communicable to non-communicable diseases, making cardiovascular disease (CVD) the leading cause of death in Europe as well as most parts of the world. According to 2017 European Cardiovascular Disease Statistics, there are more than 11 million new cases of cardiovascular disease in Europe, and cardiovascular disease accounts for $45 \%$ of all deaths. Europe is in the midst of an epidemiologic, economic, social and nutritional transition. The past few decades have shown that especially low- and middle-income countries have undergone rapid industrialization, urbanization, economic development and market globalization. Although these developments led to many positive changes in health outcomes and increased life expectancies, they all also caused inappropriate dietary patterns, physical inactivity and obesity. Fat and energy consumption is increasing mostly in Eastern Europe, and smoking remains a key public health issue despite decline in some countries.

The causal factors for CVD are well defined today. Genetic susceptibility, environmental factors and lifestyle are the most important determinants of cardiovascular health. Preventable or treatable risk factors such as high blood pressure, high cholesterol, diabetes, smoking and obesity play an important role in the development of cardiovascular disease as well as lifestyle, socioeconomic and environmental conditions. We also know that socioeconomic deprivation increases the dependence on alcohol and tobacco use as well as leading to consumption of unhealthy foods. When we look at the ranking of European countries according to GDP per capita, we see that cardiovascular risk goes hand in hand with low income.

Although genetic susceptibility is extremely important in the development of cardiovascular disease, even the subjects with high genetic risk derive benefit from healthy lifestyles. In a recent study, genetic risk was determined by a polygenic risk score of up to 50 single-nucleotide polymorphisms and adherence to a healthy lifestyle consisting of 4 factors (no current smoking, no obesity, healthy diet and regular physical activity). A favourable lifestyle was associated with a 50\% lower relative risk of $\mathrm{CAD}$ in all three groups of low, intermediate and high genetic risk (Khera et al. 2016). A healthy lifestyle will modify and decrease traditional risk factors like hypertension, diabetes, dyslipidemia and obesity. In addition lifestyle modification has positive effects beyond attenuation of traditional risk factors. To maintain cardiovascular health, it is important to strive for optimal levels of four 
health behaviours (non-smoking, body mass index $<25 \mathrm{~kg} / \mathrm{m}^{2}$, physical activity and ideal diet) and three health factors (untreated total cholesterol $<200 \mathrm{mg} / \mathrm{dL}$, untreated blood pressure $<120 /<80 \mathrm{mmHg}$ and fasting blood glucose $<100 \mathrm{mg}$ / dL) (Lloyd-Jones et al. 2010).

Although there have been tremendous advances in the medical treatment of cardiovascular risk factors in the past decades, the same cannot be said for lifestyle. The modern lifestyles have led to greater sedentary time, lower participation in active transport and time spent in leisure or purposeful physical activity, unhealthy diets and increased exposure to stress, noise and pollution.

\section{$1 \quad$ Diet}

The evidence that dietary factors influence the development of CVD derives mainly from epidemiological observations and from clinical studies on the impact of dietary changes on traditional risk factors such as plasma lipids, blood pressure or glucose levels.

In fact, epidemiological studies clearly indicate that higher consumption of fruit, non-starchy vegetables, nuts, legumes, fish, vegetable oils, yogurt and whole grains, along with a lower intake of red and processed meats and foods high in refined carbohydrates and salt, is associated with a reduced incidence of cardiovascular events. Moreover, they indicate that the replacement of animal fats, including dairy fats, with PUFAs or other vegetable sources of fats as well as fibre-rich carbohydrate foods can decrease the risk of CVD (Forouhi et al. 2018; Sacks et al. 2017).

Conversely, the evidence from randomized controlled trials (RCTs) is based on few studies that provide, in some cases, conflicting results. In this respect, the difficulties of performing clinical trials that adequately test nutritional interventions for CVD prevention cannot be underestimated since these studies require thousands of participants followed for years or decades. As to the lack of concordance between studies, this is due not only to methodological problems, particularly inadequate sample size or the short duration of many trials, but also to the difficulty of evaluating the impact of a single dietary factor independently of any other change in the diet (Forouhi et al. 2018).

These limitations suggest caution in interpreting the results of RCTs or even meta-analyses of RCTs in relation to the effect of a single dietary change on CVD, particularly where they diverge from the existing global evidence (Catapano et al. 2016). Therefore, in evaluating the role of diet in the prevention of cardiovascular diseases, we will consider not only RCTs on the impact dietary modifications on CVD events but also observational studies and clinical trials on the effects on major CVD risk factors. 
Table 1 Summary of the effects of dietary fatty acids, carbohydrates and salt on cardiovascular risk factors and cardiovascular events

\begin{tabular}{|c|c|c|c|c|c|c|c|}
\hline & $\begin{array}{l}\text { LDL- } \\
\mathrm{C}\end{array}$ & Triglycerides & $\begin{array}{l}\text { HDL- } \\
\mathrm{C}\end{array}$ & $\begin{array}{l}\text { Plasma } \\
\text { glucose }\end{array}$ & $\begin{array}{l}\text { Insulin } \\
\text { sensitivity }\end{array}$ & $\begin{array}{l}\text { Blood } \\
\text { pressure }\end{array}$ & CVD \\
\hline $\begin{array}{l}\text { Trans } \\
\text { fatty } \\
\text { acids }\end{array}$ & $\uparrow \uparrow$ & $-/ \uparrow$ & $\downarrow \downarrow$ & - & $\downarrow \downarrow$ & - & 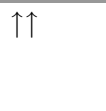 \\
\hline SAFA & $\uparrow \uparrow$ & - & $-/ \uparrow$ & - & $\downarrow \downarrow$ & $\uparrow$ & $\uparrow$ \\
\hline MUFA & - & - & - & $\downarrow$ & $\uparrow$ & $\downarrow /-$ & $\downarrow$ \\
\hline $\begin{array}{l}\text { PUFA } \\
\mathrm{n}-6\end{array}$ & - & $\downarrow$ & $\downarrow$ & $\downarrow /-$ & $\uparrow$ & $\downarrow /-$ & $\downarrow$ \\
\hline $\begin{array}{l}\text { PUFA } \\
n-3\end{array}$ & - & $\begin{array}{l}\downarrow \downarrow \text { (at high } \\
\text { dose) }\end{array}$ & - & - & - & $\begin{array}{l}\downarrow \\
\text { (at high } \\
\text { dose) }\end{array}$ & $\begin{array}{l}\downarrow \\
\text { (at high } \\
\text { dose) }\end{array}$ \\
\hline $\begin{array}{l}\text { Refined } \\
\text { starch }\end{array}$ & - & $\uparrow$ & $\downarrow$ & $\uparrow$ & $\downarrow$ & - & - \\
\hline Sugars & - & $\uparrow \uparrow$ & $\downarrow$ & $\begin{array}{l}\uparrow \text { (long } \\
\text { term) }\end{array}$ & $\downarrow \downarrow$ & $\uparrow /-$ & $\uparrow$ \\
\hline $\begin{array}{l}\text { Dietary } \\
\text { fiber }\end{array}$ & $\downarrow \downarrow$ & $-/ \downarrow$ & - & $\downarrow \downarrow$ & $\uparrow$ & $\downarrow$ & $\downarrow$ \\
\hline Salt & - & - & - & - & - & $\uparrow$ & $\uparrow$ \\
\hline
\end{tabular}

$\uparrow$ increase; $\downarrow$ decrease; - no effect

\subsection{Dietary Fat (Table 1)}

\subsubsection{Effects on CVD Risk Factors}

The available evidence supports current dietary guidelines recommending that saturated fatty acid (SAFA) intake be reduced and replaced with unsaturated fatty acids. This approach aims at improving blood lipid and lipoprotein profile in order to reduce the risk of coronary heart diseases, since high LDL cholesterol and elevated plasma triglyceride concentrations are established independent CVD risk factors (Catapano et al. 2016; Graham et al. 2012; Reiner 2013; Reiner et al. 2011). A reduction in dietary SAFA intake can be achieved through the isocaloric replacement with unsaturated fatty acids, mainly monounsaturated (MUFA, mostly cis-oleic acid) or polyunsaturated fatty acids (PUFAs) or with dietary carbohydrates. According to the results of meta-analyses of RCTs, replacing 5\% of calories from SAFA with MUFA or PUFA (predominantly linoleic acid; C18:2n-6) is able to significantly reduce LDL cholesterol levels by $0.21 \mathrm{mmol} / \mathrm{L}(8 \mathrm{mg} / \mathrm{dL})$ and $0.28 \mathrm{mmol} / \mathrm{L}(11 \mathrm{mg} / \mathrm{dL})$, respectively. In addition, this dietary approach is able to significantly improve insulin sensitivity and reduce triglyceride levels, mostly in the postprandial period; notably, the triglyceride-lowering effect is more pronounced with $n$-6 PUFA than with MUFA. As for HDL cholesterol levels, there is no relevant effect when SAFA is replaced by MUFA, whereas a small decrease occurs when they are substituted by $n-6$ PUFA (Mensink et al. 2003). Replacing SAFA with refined carbohydrates also reduces LDL cholesterol levels (by $0.16 \mathrm{mmol} / \mathrm{L}, 6.2 \mathrm{mg}$ / 
dL), but has untoward effects on other lipoproteins, namely, HDL cholesterol and plasma triglyceride levels.

Dietary trans-fatty acids (TFAs) are partially hydrogenated fatty acids formed when oils are solidified to produce margarine. Overall, the average intake of transfatty acids in western countries is presently low and derives mainly from foods of industrial origin. Quantitatively, dietary trans-fatty acids increase LDL cholesterol levels to the same extent as saturated fat, although, at variance with SAFA, they induce a marked reduction of HDL cholesterol levels (Mensink et al. 2003).

Among the various types of dietary fat, the most relevant effect on plasma triglycerides is achieved with long-chain $n-3$ polyunsaturated fatty acids; however, in order to reach an intake sufficient to induce a clinically relevant triglyceridelowering effect (usually 25-30\%), it is necessary to rely on foods artificially enriched with $n-3$ polyunsaturated fat or on pharmacological supplements. Dietary fatty acids may also affect other non-lipid CVD risk factors, particularly, blood pressure (Rasmussen et al. 2006; Zock et al. 2016). In fact, the results from the DIVAS study - a 16-week parallel group RCT on individuals with moderate CVD risk - showed that the isocaloric replacement of 9.5-9.6\% of calories from SAFA with MUFA or n-6 PUFA attenuated the increase in night systolic pressure $(-4.9 \mathrm{mmHg}, p=0.019)$ and reduced E-selectin. In line with this finding, a multicentre study in which our group was involved demonstrated that in healthy individuals, the consumption of a diet rich in MUFA for 3 months, compared to a SAFA-rich diet, significantly decreased diastolic blood pressure, provided that total fat intake was not exceedingly high (below 35-40\% energy intake) (Rasmussen et al. 2006). The beneficial effects of replacing SAFA with unsaturated fat have been summarized in a meta-analysis reporting significantly lower systolic and diastolic blood pressure with high-MUFA diets (Schwingshackl et al. 2011). As to $n$-3 PUFA, lower blood pressure levels were observed only in hypertensive subjects given $>3 \mathrm{~g} /$ day as pharmacological supplement (Miller et al. 2014).

Dietary fatty acids are also able to influence insulin sensitivity and, more in general, glucose metabolism; this was suggested by a recent meta-analysis of RCTs indicating that increased PUFA intake is able to improve long-term glycaemic control and reduce insulin resistance (Imamura et al. 2016). In particular, replacing $5 \%$ of calories from SAFA partially replacing SAFA or carbohydrates with PUFA or MUFA improved both blood glucose control and insulin sensitivity; moreover, PUFA increased also insulin secretion (Imamura et al. 2016). Other clinical trials have shown that the isocaloric replacement of SAFA with MUFA or PUFA improved insulin sensitivity in healthy subjects (Vessby et al. 2001) and also reduced hepatic fat accumulation (Summers et al. 2002). In line with these findings, Bozzetto and colleagues showed that partially replacing SAFA and carbohydrates with MUFA for an 8-week period induced a clinically relevant reduction of hepatic fat content (29\%) in type 2 diabetic patients (Bozzetto et al. 2012).

\subsubsection{Effects on CVD Events}

TFAs represent one of the few dietary components unanimously considered as deleterious in relation to the cardiovascular risk. Data in the literature are very 
consistent in showing that TFA intake is associated with a higher risk of CVD and sudden death; this relationship is stronger than with any other nutrient (Mozaffarian et al. 2006). In fact, a meta-analysis of four prospective cohort studies has shown that a $2 \%$ increase in energy intake from TFAs is associated with a $23 \%$ higher incidence of coronary heart disease (CHD) (Mozaffarian et al. 2006).

In recent years, concern has been expressed regarding the impact of dietary saturated fatty acids (SFAs) on cardiovascular risk. The uncertainty stems from recent meta-analyses failing to find an association between the amount of SFA in the habitual diet and the incidence of CVD (Chowdhury et al. 2014; Siri-Tarino et al. 2010). However, other meta-analyses of prospective studies and RCTs as well as large longitudinal observations clearly indicate that high SFA is linked with a small but potentially important increase in cardiovascular risk (Jakobsen et al. 2009; Li et al. 2015); recently, a Cochrane systematic review has shown that a lowsaturated-fat diet induces a $17 \%$ reduction in cardiovascular events compared to a control diet (Hooper et al. 2015).

Inconsistencies are due to multiple reasons and, in particular, to the way the data are evaluated; in fact, the meta-analysis by Siri-Tarino overadjusted the outcomes by correcting them for plasma lipid levels, thus eliminating one of the major pathophysiological links between SFA intake and CVD (Siri-Tarino et al. 2010). More in general, in RCTs evaluating the effects of SFAs on CVD, the choice of the foods/ nutrients utilized in the control diet to replace SFAs (for instance, unsaturated fat versus refined grains) can influence the effect observed and significantly modify the outcomes of the comparison. The importance of SFA as a risk factor for CVD has been recently underlined by recommendations of scientific societies and public health authorities (Sacks et al. 2017; USDA 2016).

PUFA intake is associated with lower CVD risk, as shown in a meta-analysis of 13 prospective cohort studies $(310,602$ subjects) reporting that dietary linoleic acid (LA) intake is inversely associated with the risk of CHD incidence (15\%) and mortality (21\%) (Farvid et al. 2014).

The available data on the relationship between dietary cholesterol and CVD are inconsistent, probably due to the difficulty to evaluate the impact of dietary cholesterol independently of SAFA that is present in many cholesterol-rich foods. However, a recent meta-analysis from six prospective US cohorts in which many of the possible confounders were properly accounted for showed that each additional $300 \mathrm{mg}$ of dietary cholesterol consumed per day (roughly one egg) was significantly associated with higher risk of CVD incidence (17\%) and mortality (18\%) (Zhong et al. 2019).

\subsection{Dietary Carbohydrates (Table 1)}

\subsubsection{Effects on CVD Risk Factors}

Dietary carbohydrates are the main determinants of postprandial blood glucose levels, which represent an important and independent risk factor for cardiovascular diseases, not only in diabetic patients but also in individuals with normal fasting 
glucose values (Rivellese et al. 2012). There is a wealth of data indicating that a high intake of refined carbohydrates affects also plasma insulin and triglyceride levels, both at fasting and in the postprandial period, as well as HDL-cholesterol levels (Katan et al. 1997; Riccardi and Rivellese 1991; Sacks and Katan 2002). However, it is worth highlighting that dietary carbohydrates are a heterogeneous class of nutrients that include not only sugars and refined starches but also dietary fibre (i.e. non-starch polysaccharides), with different chemical structures and physical forms and therefore different metabolic effects in relation to their digestion and intestinal activity (Giacco et al. 2016). In line with this concept is the recent evidence supporting the hypothesis that the quality of dietary carbohydrates rather than their amount plays a relevant role in the prevention and development of major cardiovascular risk factors (Reynolds et al. 2019; Riccardi and Costabile 2019). The main indicators of carbohydrate quality refer essentially to the amount of fibre and the glycaemic index of carbohydrate-rich foods as well as the sugar content.

There is consolidated evidence of the beneficial role of consuming low-glycaemic index carbohydrates on blood glucose control, HbA1c levels, fasting and postprandial triglyceride levels and HDL cholesterol levels (Augustin et al. 2015; Riccardi et al. 2008; Thomas and Elliott 2010). However, the best available evidence of the beneficial metabolic impact of low-glycaemic index foods comes from studies in people with type 2 diabetes.

In the last decades, several clinical trials have been conducted to investigate the possible effects of different types of fibre - i.e. fibre from whole grain, legumes, fruit and vegetables and fibre supplements - on body weight changes, blood glucose metabolism, plasma lipids and blood pressure control. The overall evidence shows that soluble fibre, mainly $\beta$-glucans from oat and barley but also inulin, guar gum, glucomannan, pectin and psyllium principally found in fruit, vegetables and legumes, are able to significantly reduce plasma LDL-cholesterol levels, improve blood pressure (mainly $\beta$-glucan and psyllium) and induce a small reduction in body weight (Bozzetto et al. 2018). Moreover, there is evidence of a triglyceride-lowering effect of dietary fibre and whole grain during the postprandial period (Bozzetto et al. 2014; De Natale et al. 2009; Giacco et al. 2014).

These results support the current nutritional recommendations to replace refined grains with whole grains and increase dietary fibre intake to at least $25-29 \mathrm{~g}$ per day to reduce the incidence of the main cardiometabolic risk factors.

Among refined carbohydrates, specific attention should be paid to simple sugars, particularly fructose, whose adverse effects (at amounts higher than $10 \%$ of total energy) on human health have been highlighted. According to evidence from observational and intervention studies, the consumption of high fructose-sweetened beverages increases fasting and postprandial triglycerides levels, especially in subjects with obesity and hypertriglyceridemia (Chiavaroli et al. 2015; Stanhope et al. 2009), and also has adverse effects on visceral fat deposition, blood pressure and insulin sensitivity (Stanhope et al. 2009). 


\subsubsection{Effects on CVD Events}

Since dietary carbohydrates are a heterogeneous class of nutrients with different metabolic effects, the evaluation of the relationship between the total amount of carbohydrates in the diet and the incidence of cardiovascular diseases is misleading. In fact, a recent prospective study in 135, 335 individuals from 613 communities in 18 countries has shown, in contrast with the results from meta-analyses of several large cohort studies in North America and Europe, that a high carbohydrate intake is associated with higher risk of total mortality (Dehghan et al. 2017). The reasons for the inconsistency have been highlighted by a recent meta-analysis performed on data from four US communities showing that both very high and very low carbohydrate diets were associated with increased mortality: the lowest mortality was observed in people in whom carbohydrates represented $50-55 \%$ of their energy intake (Seidelmann et al. 2018). However, the quality of carbohydrate-rich foods rather than their quantity has the strongest effect on the development of major health outcomes; in this respect, what really matters is the presence of vegetable fibre and/or sugar in the diet.

In fact, a recent meta-analysis reports a $15-30 \%$ decrease in all-cause and cardiovascular-related mortality and in the incidence of coronary heart disease between the highest and the lowest dietary fibre intake. The relationship between fibre intake and CVD was linear although the greatest risk reductions were observed for individuals with a fibre intake of 25-29 g/day, mainly provided by cereal fibre; a habitual consumption of 40-50 g/day of whole grains was associated with a risk reduction of 20-30\% (Reynolds et al. 2019).

On the other hand, a higher consumption of sugar is associated with an increased risk of coronary events (17\% for every serving/day increase in sugar-sweetened beverages consumption) (Xi et al. 2015).

\subsection{Salt (Table 1)}

\subsubsection{Effects on CVD Risk Factors}

Extensive scientific evidence shows that reducing dietary salt (sodium chloride) intake significantly decreases systolic and diastolic blood pressure in adults with or without hypertension (He et al. 2013; Stamler et al. 2018). Recent data from the international multicentre population INTERMAP study reported a positive association between salt intake and blood pressure values also within the normal range (Zhou et al. 2019). In addition to sodium reduction, an increase in potassium intake is beneficial to prevent and control blood pressure in people with hypertension, with no adverse effects on plasma lipid concentrations (Aburto et al. 2013a). In line with these findings, current nutritional recommendations include a potassium intake of at least $3.5 \mathrm{~g}$ per day in adults. Therefore, a diet that combines low sodium and high potassium intakes is more effective in reducing blood pressure and hypertension risk. An example of this type of approach is the DASH diet, recommended for the non-pharmacological management of hypertension. This diet includes also changes in the quality of fat - promoting unsaturated fat and carbohydrates - favouring 
unrefined ones. Recent results from a systematic review and meta-analyses of RCTs support the beneficial effect of the DASH diet not only on blood pressure but also on other cardiovascular risk factors, including total and LDL cholesterol concentrations (Siervo et al. 2015).

\subsubsection{Effects on CVD Events}

Two recent meta-analyses of cohort studies showed a direct relationship between sodium intake and stroke, CVD and CHD mortality; a higher sodium intake was significantly associated with an increased risk of CVD (12\%) and CHD (32\%) mortality, in addition to a much higher risk of stroke mortality (63\%) (Aburto et al. 2013b; Poggio et al. 2015). Consistently, two recent meta-analyses of clinical trials showed that dietary salt reduction induces a significantly lower incidence (-20\%) of CVD (Adler et al. 2014; He and MacGregor 2011).

Actually, in many parts of the world, the average sodium consumption (mainly derived from dietary salt intake and food additives, such as sodium glutamate) is above that recommended by guidelines: less than $5 \mathrm{~g}$ of salt/day (equivalent to about $2.3 \mathrm{~g}$ of sodium daily) (WHO Guidelines Approved by the Guidelines Review Committee 2012).

In the last decades, the science of human nutrition has shifted from a reductionistic approach focused on specific nutrients to a broader view emphasizing the role of food groups/dietary patterns (van Horn et al. 2016). This paradigm change is due to convincing scientific evidence showing that human health is indeed influenced by single nutrients but also by their complex interactions and by their interplay with other bioactive substances present in foods. These are likely to act synergistically, and, therefore, their impact on human health may not be appreciated unless evaluated within the context of the whole diet. Furthermore, characteristics other than nutrient combinations (i.e. physical features of the foods, technological processes, cooking procedures) may influence the absorption and bioavailability of nutrients and in turn modulate their metabolic effects. Therefore, there is growing attention to the identification of dietary patterns associated to the risk of disease or death. The Mediterranean diet is one of dietary patterns for which strong evidence from observational and intervention studies has accumulated on the benefits in the primary and secondary prevention of cardiovascular disease and other major chronic diseases, such as type 2 diabetes, cancer and probably cognitive impairment (de Lorgeril et al. 1999; Estruch et al. 2018; Sofi et al. 2014).

The Mediterranean diet is characterized by a food pattern resembling the healthy food choices outlined above and, in particular, by the regular consumption of plant foods - including vegetables, pulses, nuts, fruits and unrefined cereals - and fish as well as a low intake of red and processed meats and whole fat dairy products; the main source of fat is extra virgin olive oil (Table 1). The optimal nutrient distribution and the balanced food choices of this dietary pattern have supported it as a useful model for the implementation of a healthy diet at the population level. Its strengths are not only the evidence in support of its beneficial impact on cardiovascular disease prevention but also its very deep cultural roots that are the source of the large body of 
Table 2 Summary of lifestyle measures and healthy food choices for managing total cardiovascular risk

Dietary recommendations should always take into account local food habits; however, interest in healthy food choices from other cultures should be promoted

A wide variety of foods should be eaten. Energy intake should be adjusted to prevent overweight and obesity

Consumption of fruits, vegetables, legumes, nuts, wholegrain cereal foods and fish (especially oily) should be encouraged

Foods rich in trans-fatty acids should be avoided totally; foods rich in SFAs (tropical oils, fatty or processed meat, sweets, cream, butter, regular cheese) should be replaced with the above foods and with monounsaturated fat (extra virgin olive oil) and polyunsaturated fat (non-tropical vegetable oils) in order to keep SFA intake of $<10 \mathrm{En} \%$ ( $<7 \%$ in the presence of high plasma cholesterol values)

Salt intake should be reduced to $<5 \mathrm{~g} /$ day by avoiding table salt and limiting salt in cooking and by choosing fresh or frozen unsalted foods; many processed and convenience foods, including bread, are high in salt

For those who drink alcoholic beverages, moderation should be advised ( $<10 \mathrm{~g} /$ day for women and $<20 \mathrm{~g} /$ day for men), and patients with hypertriglyceridaemia should abstain

The intake of beverages and foods with added sugars, particularly soft drinks, should be limited, especially for persons who are overweight, have hypertriglyceridaemia, metabolic syndrome or diabetes

Physical activity should be encouraged, aiming at regular physical exercise for at least $30 \mathrm{~min} / \mathrm{day}$ every day

Use of and exposure to tobacco products should be avoided

culinary recipes to make a healthy choice a gastronomical adventure (Catapano et al. 2016) (Table 2).

\section{$2 \quad$ Physical Activity}

Having a sedentary life is a cardiovascular risk factor in itself. The amount of time spent being sedentary is increasing all over Europe even in young people. Watching $\mathrm{TV}$, being stationary in front of a computer and using vehicles for transportation are the disadvantages of modern living. Especially in middle-aged females, sedentary lifestyle is almost as risky as smoking (Brown et al. 2015). Several studies have shown that sedentary behaviour increases cardiovascular risk. One of the largest prospective registries was the PURE study where physical activity was recorded in 130, 843 participants without existing CVD for 6.9 years. Compared with low physical activity $(<600$ metabolic equivalents $[\mathrm{MET}] \times$ minutes per week or $<150$ min per week of moderate-intensity physical activity), moderate (600-3,000 MET $\times$ minutes or 150-750 min per week) and high physical activity $(>3,000$ MET $\times$ minutes or $>750$ min per week) were associated with graded reduction in mortality and major cardiovascular events (Lear et al. 2017).

Experimental studies that mimicked sedentary behaviour have shown greater postprandial glucose and insulin levels during bouts of prolonged sitting compared 
with individuals taking frequent standing or walking breaks (Pulsford et al. 2017). Compared with prolonged sitting, breaking up sitting time with intermittent, even light-intensity activity, can increase expression of anti-inflammatory and antioxidative pathway modulators (Latouche et al. 2013). A recent meta-analysis investigated the association between sedentary behaviour and incident CVD events using data from nine prospective cohort studies and showed that risk increased with increased sedentary time, with the highest risk being in more than $10 \mathrm{~h}$ a day of sedentary time (Pandey et al. 2016). A recent study has shown that sustained physical activity but not weight loss was associated with improved survival in coronary heart disease (Moholdt et al. 2018).

Physical activity has a positive effect on body weight, blood pressure, blood glucose, lipid levels, endothelial function, autonomous regulation and coagulation. The summary of all these effects leads to a decrease in cardiovascular disease incidence and mortality. Physical activity has both acute and chronic effects on blood pressure. With increased physical activity, blood pressure is lowered especially in subjects with prehypertension compared to those with normal blood pressure (Physical Activity Guidelines Advisory Committee 2018). Physical activity combined with calorie restriction can contribute to weight loss and supports the maintenance of weight loss. The risk of type 2 diabetes mellitus is greatly reduced with physical activity, and this benefit is observed irrespective of body weight. Engaging in 150-300 min a week of moderate-intensity physical activity can reduce the risk of developing type 2 diabetes mellitus by 25-35\% (Physical Activity Guidelines Advisory Committee 2018). Regular physical activity or exercise can affect serum lipid levels favourably. Men and women who exercise regularly have significantly lower LDL-C and VLDL and higher HDL-C levels compared to ageand gender-matched sedentary controls (Vodak et al. 1980; Wood et al. 1976). Similarly, moderate physical exercise decreases LDL-C and triglycerides and increases HDL in male survivors of myocardial infarction (Ballantyne et al. 1982). The effect of exercise on lipids varies in exercise intervention studies, and there appears to be a minimum exercise volume to increase HDL-C (Kodama et al. 2007). There are additional antiatherogenic effects of physical activity besides those affecting traditional cardiovascular disease risk factors (Green et al. 2017). Exercise intensity improves vascular endothelial function in a dose-dependent manner for aerobic activities (Ashor et al. 2015). Endothelial function is improved with exercise even in the absence of changes in classical risk factors like lipids levels, blood pressure, glucose or BMI (Green et al. 2008). Acute- and moderate-intensity exercises increase shear stress, which stimulates endothelium-dependent vasodilatation through the increased synthesis of nitric oxide (Erkens et al. 2017). Regular exercise training also leads to increased coronary artery size and dilatation capacity (Haskell et al. 1993) and increased luminal diameter of conduit arteries (Green et al. 2008). Exercise training can reduce the wall thickness of conduit arteries and increases the development of coronary collateral blood vessels (Thijssen et al. 2012). Unhealthy gut microbiota has recently been shown to increase the risk of CVD (Fu et al. 2015; Lanter et al. 2014). Regular physical activity or endurance exercise training can positively alter the human gut microbiota by increasing 
bacterial diversity, increasing faecal concentrations of short-chain fatty acids and increasing the proportion of healthy bacterial species (Allen et al. 2018; Bressa et al. 2017; Estaki et al. 2016). A recent study on endurance training has shown that exercise increases telomerase activity and length, whereas resistance training did not show these effects (Werner et al. 2019). Ideally, a combination of resistance and endurance training should be implemented (Reiner et al. 2019).

Physical activity is any activity that moves the skeletal muscles and consumes energy, whereas systematic physical activity, like swimming and running, is described as exercise. In healthy individuals, regular physical activity decreases all-cause and cardiovascular mortality by 20-30\% (Lollgen et al. 2009; Moore et al. 2012; Sattelmair et al. 2011). Regular physical activity increases cardiorespiratory fitness (The American College of Sports Medicine 2014). Cardiorespiratory fitness is associated with reduced prevalence of CVD risk factors and improves prognosis (Harber et al. 2017). A meta-analysis of 33 studies in over 100, 000 individuals observed that every 1 estimated MET increase in CRF was associated with 13 and 15\% reductions in all-cause and CVD mortality (Nauman et al. 2017). Cardiorespiratory fitness is important even in the presence of other risk factors and shown to be protective even in men with metabolic syndrome (Katzmarzyk et al. 2004) and obesity (Moholdt et al. 2017).

Although all agree that any level of physical activity is better than none, there is controversy about the optimal dose of physical activity for CVD prevention. The intensity and frequency of exercise should be personalized and adapted to a person's needs as well as lifestyle. We also need to keep in mind that there is a high degree of interindividual variation in cardiorespiratory fitness responses to exercise.

The 2016 ESC prevention guideline recommends (Piepoli et al. 2016) for healthy adults of all ages to perform at least $150 \mathrm{~min}$ a week of moderate-intensity or $75 \mathrm{~min}$ a week of vigorous-intensity aerobic physical activity (PA) or an equivalent combination. For additional benefits in healthy adults, a gradual increase in aerobic PA to $300 \mathrm{~min}$ a week of moderate-intensity or $150 \mathrm{~min}$ a week of vigorous-intensity aerobic PA, or an equivalent combination thereof, is recommended. Multiple sessions of PA should be considered, each lasting $\geq 10 \mathrm{~min}$ and evenly spread throughout the week. Clinical evaluation, including exercise testing, should be considered for sedentary people with cardiovascular risk factors who intend to engage in vigorous PAs or sports.

Even low-level physical activity, such as low-dose running or commuting to work by bicycle, has been associated with a lower incidence of obesity, arterial hypertension, dyslipidaemia and diabetes mellitus (Grontved et al. 2016). The most recent Physical Activity Guidelines for Americans from the US Department of Health and Human Services has stated that benefits related to physical activity start even earlier and are easier to obtain than was previously thought according to recent evidence (Physical Activity Guidelines Advisory Committee 2018). These guidelines state that although one should try to get to guideline-recommended goals, the threshold at which health benefits begin to start is less than 150 min a week for most outcomes. There is no lower limit to the benefits of physical activity in reducing cardiovascular disease risk. However, getting to goal helps in obtaining benefits for the greatest 
number of outcomes. Additional physical activity confers additional benefits, and health risk does not seem to increase with high amounts of physical activity, even beyond 3-5 times the $150 \mathrm{~min}$ a week recommendation in healthy individuals. Compared with inactive adults, meeting the goals is associated with a $14 \%$ reduced risk of developing coronary heart disease (Physical Activity Guidelines Advisory Committee 2018).

2019 ACC/AHA Guideline on the Primary Prevention of Cardiovascular Disease (Arnett et al. 2019) also recommends that adults should engage in at least $150 \mathrm{~min}$ per week of accumulated moderate-intensity or 75 min per week of vigorousintensity aerobic physical activity to reduce atherosclerotic cardiovascular disease. For adults unable to meet the minimum physical activity recommendations, engaging in some moderate- or vigorous-intensity physical activity, even if less than this recommended amount, and decreasing sedentary behaviour can be beneficial to reduce CVD risk.

Although some exercise is beneficial for all, for those with known cardiovascular disease, the dose of exercise needs to be individualised to prevent adverse outcomes. The risk of having an adverse cardiovascular event during exercise is very low, especially during light exercise. However, it is best to do a risk assessment and tailor the exercise program according to the need of the individual. Light- and moderateintensity exercise can be done with minimal risk, whereas intensive exercise should be preceded by a medical evaluation, especially for middle-aged people. The cardioprotective effects of regular PA, whether performed in low or high volumes, are clear and extend across all ages, gender and race (El Saadany et al. 2017; Nes et al. 2017; O'Donovan et al. 2018). Whether overexercising in healthy individuals can have unfavourable consequences has been questioned. In some studies, longterm strenuous exercise has been shown to increase the risk of atrial fibrillation (Gorenek Chair et al. 2017). It is never too late to start exercising. The Aerobics Center Longitudinal Study has shown that men who were in the lowest percentile of $\mathrm{CRF}$ at their first examination but fit at the time of their second examination years later had a 52\% reduction in CVD mortality compared with men who remained unfit (Blair et al. 1995). For every one estimated MET increase, all-cause and CVD mortalities were reduced by $15 \%$ and 19\%, respectively (Lee et al. 2011).

\section{$3 \quad$ Smoking}

Smoking is one of the most important modifiable risk factors for CVD. Furthermore, stopping smoking is the most cost-effective prevention intervention. It is well known that smoking and using all forms of tobacco products cause CVD and increase mortality (Mons et al. 2015; Teo et al. 2006). Because tobacco use even in small amounts increases cardiovascular risk, reducing the number of cigarettes or the amount of tobacco consumption is not sufficient. Even smoking one cigarette per day carries a risk (Hackshaw et al. 2018). Therefore there is no safe level of smoking and quitting is mandatory. Passive smoking also increases cardiovascular risk, and 
studies have shown that second-hand smoke exposure increases CVD and triggers adverse events in non-smokers (Lv et al. 2015).

Cigarette smoke contains more than 4,000 chemicals and toxins like nicotine, carbon monoxide, cadmium and oxidants (Smith and Fischer 2001). Exposure to these chemicals affects all stages of atherosclerotic vascular disease unfavourably. Endothelial dysfunction due to reduced NO bioavailability and activation of NF-kB will lead to functional and eventually physical damage to the endothelium (Collins 1993; Rahman and Laher 2007). Nicotine has been shown to promote MMP expression in smooth muscle cells and decreased collagen synthesis in the arterial wall impairing the stability of the plaque (Carty et al. 1996; Raveendran et al. 2004) Cigarette smoke increases systemic inflammation (Csordas et al. 2008). The catecholamines are increased in the circulation triggering vasospasm (Zhu and Parmley 1995). Smoking also alters the balance between pro- and anticoagulant factors towards procoagulation. Plaque thrombogenicity is increased with increased plasma tissue factor, VWF, thrombin and thrombomodulin and activated platelets (Markuljak et al. 1995; Miller et al. 1998). Upon smoking cessation, several of these prothrombotic changes revert to normal. Blood viscosity is also increased with smoking (Lowe et al. 1980). All of these effects contribute to plaque formation, vulnerability and thrombus development. Interestingly, there is a genetic susceptibility to the atherothrombotic effects of smoking. Different variants have been described that alter one's susceptibility to the negative effects of smoking (Wang et al. 1996).

Smoking dependence is a chronic disease that needs to be managed with patience, expertise and time. The ESC prevention guidelines recommend the following strategy for smoking cessation (Piepoli et al. 2016): systematically inquire about smoking status at every opportunity; unequivocally urge all smokers to quit; determine the person's degree of addiction and readiness to quit; agree on a smoking cessation strategy, including setting a quit date, behavioural counselling and pharmacological support; and arrange a schedule of follow-up ("five As" for a smoking cessation strategy for routine practice - ask, advise, assess, assist, and arrange). In case where advice and motivational interventions fail, drug-based interventions can be used to aid quitting smoking. Nicotine replacement therapies, bupropion and varenicline, have been shown to be helpful in increasing quitting (Cahill et al. 2013; Hughes et al. 2014; Stead et al. 2012). Electronic cigarettes emitting an aerosol containing nicotine have been developed to aid quitting; however, they may also emit toxic gases that may increase the risk of cardiovascular and pulmonary diseases, arrhythmias and hypertension (Benowitz and Fraiman 2017). Patients often are concerned about weight gain after smoking cessation. However a recent study from the cohorts of the Nurses' Health Study (NHS), (NHS II) and the Health Professionals Follow-Up Study (HPFS) has shown that even if the smoking cessation was accompanied by substantial weight gain, this did not decrease the benefits of quitting smoking on reducing cardiovascular and all-cause mortality and cardiovascular mortality decreased in all weight-change groups (Hu et al. 2018).

Modern lifestyles expose us to air pollution and occupational noise which also have a negative impact on cardiovascular diseases. It has been shown that the risk of 
STEMI rises within hours of exposure to air pollutants (Sahlen et al. 2019). Furthermore, several investigations support that inhalation of ambient particulate matter triggers pulmonary and systemic inflammation resulting in metabolic syndrome and cardiopulmonary disease (Clementi et al. 2019). Exposure to high occupational noise is also associated with increase in hypertension, cardiovascular disease and cardiovascular mortality (Skogstad et al. 2016; Teixeira et al. 2019).

A healthy lifestyle is critically important to improve cardiovascular health and increase the control of risk factors. Healthy lifestyles should be adapted from birth to childhood and maintained throughout the lifespan. This can be possible only with creating health-promoting environments and improving healthcare policies, the interaction between the patient and the physician and education of the patient and the public about healthy lifestyles.

The WHO has put together a global action plan for the prevention and control of non-communicable diseases (WHO 2013). The goal is to reduce the risk of premature non-communicable disease deaths by $25 \%$ by 2025 . According to this plan, reductions in tobacco use, salt intake, physical inactivity, harmful use of alcohol, raised blood pressure as well as stopping the diabetes and obesity epidemics are the goals. Early action and aggressive implementation of this plan may decrease the cardiovascular disease epidemic if the individual WHO targets are met and healthcare systems are strengthened. At the individual level, it is important for us to promote healthy lifestyles to everyone, including patients who already have CVD since it is never too late to benefit from a healthy lifestyle.

\section{References}

Aburto NJ, Hanson S, Gutierrez H, Hooper L, Elliott P, Cappuccio FP (2013a) Effect of increased potassium intake on cardiovascular risk factors and disease: systematic review and metaanalyses. BMJ (Clin Res Ed) 346:f1378. https://doi.org/10.1136/bmj.f1378

Aburto NJ, Ziolkovska A, Hooper L, Elliott P, Cappuccio FP, Meerpohl JJ (2013b) Effect of lower sodium intake on health: systematic review and meta-analyses. BMJ (Clin Res Ed) 346:f1326. https://doi.org/10.1136/bmj.f1326

The American College of Sports Medicine (2014) ACSM's guidelines for exercise testing and prescription. 9th edn. Wolters Kluwer/Lippincott Williams \& Wilkins, Philadelphia, PA

Adler AJ, Taylor F, Martin N, Gottlieb S, Taylor RS, Ebrahim S (2014) Reduced dietary salt for the prevention of cardiovascular disease. Cochrane Database Syst Rev 7:Cd009217. https://doi.org/ 10.1002/14651858.CD009217.pub3

Allen JM et al (2018) Exercise alters gut microbiota composition and function in lean and obese humans. Med Sci Sports Exerc 50:747-757. https://doi.org/10.1249/mss.0000000000001495

Arnett DK et al (2019) 2019 ACC/AHA guideline on the primary prevention of cardiovascular disease circulation. https://doi.org/10.1161/cir.0000000000000678

Ashor AW, Lara J, Siervo M, Celis-Morales C, Oggioni C, Jakovljevic DG, Mathers JC (2015) Exercise modalities and endothelial function: a systematic review and dose-response metaanalysis of randomized controlled trials sports medicine (Auckland, NZ). Sports Med 45:279-296. https://doi.org/10.1007/s40279-014-0272-9

Augustin LS et al (2015) Glycemic index, glycemic load and glycemic response: An International Scientific Consensus Summit from the International Carbohydrate Quality Consortium (ICQC). Nutr Metab Cardiovasc Dis 25:795-815. https://doi.org/10.1016/j.numecd.2015.05.005 
Ballantyne FC, Clark RS, Simpson HS, Ballantyne D (1982) High density and low density lipoprotein subfractions in survivors of myocardial infarction and in control subjects. Metab Clin Exp 31:433-437

Benowitz NL, Fraiman JB (2017) Cardiovascular effects of electronic cigarettes. Nat Rev Cardiol 14:447-456. https://doi.org/10.1038/nrcardio.2017.36

Blair SN, Kohl HW 3rd, Barlow CE, Paffenbarger RS Jr, Gibbons LW, Macera CA (1995) Changes in physical fitness and all-cause mortality. A prospective study of healthy and unhealthy men. JAMA 273:1093-1098

Bozzetto L et al (2012) Liver fat is reduced by an isoenergetic MUFA diet in a controlled randomized study in type 2 diabetic patients. Diabetes Care 35:1429-1435. https://doi.org/10. 2337/dc12-0033

Bozzetto L et al (2014) A CHO/fibre diet reduces and a MUFA diet increases postprandial lipaemia in type 2 diabetes: no supplementary effects of low-volume physical training. Acta Diabetol 51:385-393. https://doi.org/10.1007/s00592-013-0522-6

Bozzetto L et al (2018) Dietary fibre as a unifying remedy for the whole spectrum of obesityassociated cardiovascular risk. Nutrients 10. https://doi.org/10.3390/nu10070943

Bressa C et al (2017) Differences in gut microbiota profile between women with active lifestyle and sedentary women. PLoS One 12:e0171352. https://doi.org/10.1371/journal.pone.0171352

Brown WJ, Pavey T, Bauman AE (2015) Comparing population attributable risks for heart disease across the adult lifespan in women. Br J Sports Med 49:1069-1076. https://doi.org/10.1136/ bjsports-2013-093090

Cahill K, Stevens S, Perera R, Lancaster T (2013) Pharmacological interventions for smoking cessation: an overview and network meta-analysis. Cochrane Database Syst Rev 5:Cd009329. https://doi.org/10.1002/14651858.CD009329.pub2

Carty CS, Soloway PD, Kayastha S, Bauer J, Marsan B, Ricotta JJ, Dryjski M (1996) Nicotine and cotinine stimulate secretion of basic fibroblast growth factor and affect expression of matrix metalloproteinases in cultured human smooth muscle cells. J Vasc Surg 24:927-934

Catapano AL et al (2016) 2016 ESC/EAS guidelines for the management of dyslipidaemias. Eur Heart J 37:2999-3058. https://doi.org/10.1093/eurheartj/ehw272

Chiavaroli L et al (2015) Effect of fructose on established lipid targets: a systematic review and meta-analysis of controlled feeding trials. J Am Heart Assoc 4:e001700. https://doi.org/10. 1161/jaha.114.001700

Chowdhury R et al (2014) Association of dietary, circulating, and supplement fatty acids with coronary risk: a systematic review and meta-analysis. Ann Intern Med 160:398-406. https://doi. org/10.7326/m13-1788

Clementi EA et al (2019) Metabolic syndrome and air pollution: a narrative review of their cardiopulmonary effects. Toxics 7. https://doi.org/10.3390/toxics7010006

Collins T (1993) Endothelial nuclear factor-kappa B and the initiation of the atherosclerotic lesion. Lab Invest 68:499-508

Physical Activity Guidelines Advisory Committee (2018) 2018 Physical Activity Guidelines Advisory Committee Scientific Report. Washington, DC

Csordas A, Wick G, Laufer G, Bernhard D (2008) An evaluation of the clinical evidence on the role of inflammation and oxidative stress in smoking-mediated cardiovascular disease. Biomark Insights 3:127-139

de Lorgeril M, Salen P, Martin JL, Monjaud I, Delaye J, Mamelle N (1999) Mediterranean diet, traditional risk factors, and the rate of cardiovascular complications after myocardial infarction: final report of the Lyon diet heart study. Circulation 99:779-785. https://doi.org/10.1161/01.cir. 99.6.779

de Natale C et al (2009) Effects of a plant-based high-carbohydrate/high-fiber diet versus highmonounsaturated fat/low-carbohydrate diet on postprandial lipids in type 2 diabetic patients. Diabetes Care 32:2168-2173. https://doi.org/10.2337/dc09-0266 
Dehghan $\mathrm{M}$ et al (2017) Associations of fats and carbohydrate intake with cardiovascular disease and mortality in 18 countries from five continents (PURE): a prospective cohort study. Lancet 390:2050-2062. https://doi.org/10.1016/s0140-6736(17)32252-3

el Saadany T, Richard A, Wanner M, Rohrmann S (2017) Sex-specific effects of leisure-time physical activity on cause-specific mortality in NHANES III. Prev Med 101:53-59. https://doi. org/10.1016/j.ypmed.2017.05.029

Erkens R, Suvorava T, Kramer CM, Diederich LD, Kelm M, Cortese-Krott MM (2017) Modulation of local and systemic Heterocellular communication by mechanical forces: a role of endothelial nitric oxide synthase. Antioxid Redox Signal 26:917-935. https://doi.org/10.1089/ars.2016. 6904

Estaki M et al (2016) Cardiorespiratory fitness as a predictor of intestinal microbial diversity and distinct metagenomic functions. Microbiome 4:42. https://doi.org/10.1186/s40168-016-0189-7

Estruch R et al (2018) Primary prevention of cardiovascular disease with a Mediterranean diet supplemented with extra-virgin olive oil or nuts. N Engl J Med 378:e34. https://doi.org/10.1056/ NEJMoa1800389

Farvid MS et al (2014) Dietary linoleic acid and risk of coronary heart disease: a systematic review and meta-analysis of prospective cohort studies. Circulation 130:1568-1578. https://doi.org/10. 1161/circulationaha.114.010236

Forouhi NG, Krauss RM, Taubes G, Willett W (2018) Dietary fat and cardiometabolic health: evidence, controversies, and consensus for guidance. BMJ (Clin Res Ed) 361:k2139. https://doi. org/10.1136/bmj.k2139

Fu J et al (2015) The gut microbiome contributes to a substantial proportion of the variation in blood lipids. Circ Res 117:817-824. https://doi.org/10.1161/circresaha.115.306807

Giacco R et al (2014) A whole-grain cereal-based diet lowers postprandial plasma insulin and triglyceride levels in individuals with metabolic syndrome Nutrition, metabolism, and cardiovascular diseases. Nutr Metab Cardiovasc Dis 24:837-844. https://doi.org/10.1016/j.numecd. 2014.01.007

Giacco R, Costabile G, Riccardi G (2016) Metabolic effects of dietary carbohydrates: the importance of food digestion. Food Res Int 88:336-341

Gorenek Chair B et al (2017) European Heart Rhythm Association (EHRA)/European Association of Cardiovascular Prevention and Rehabilitation (EACPR) position paper on how to prevent atrial fibrillation endorsed by the Heart Rhythm Society (HRS) and Asia Pacific Heart Rhythm Society (APHRS). Eur J Prev Cardiol 24:4-40. https://doi.org/10.1177/2047487316676037

Graham I, Cooney MT, Bradley D, Dudina A, Reiner Z (2012) Dyslipidemias in the prevention of cardiovascular disease: risks and causality. Curr Cardiol Rep 14:709-720. https://doi.org/10. 1007/s11886-012-0313-7

Green DJ, O'Driscoll G, Joyner MJ, Cable NT (2008) Exercise and cardiovascular risk reduction: time to update the rationale for exercise? J Appl Physiol 105:766-768. https://doi.org/10.1152/ japplphysiol.01028.2007

Green DJ, Hopman MT, Padilla J, Laughlin MH, Thijssen DH (2017) Vascular adaptation to exercise in humans: role of hemodynamic stimuli. Physiol Rev 97:495-528. https://doi.org/10. 1152/physrev.00014.2016

Grontved A et al (2016) Bicycling to work and primordial prevention of cardiovascular risk: a cohort study among Swedish men and women. J Am Heart Assoc 5. https://doi.org/10.1161/ jaha.116.004413

Hackshaw A, Morris JK, Boniface S, Tang JL, Milenkovic D (2018) Low cigarette consumption and risk of coronary heart disease and stroke: meta-analysis of 141 cohort studies in 55 study reports. BMJ (Clin Res Ed) 360:j5855. https://doi.org/10.1136/bmj.j5855

Harber MP, Kaminsky LA, Arena R, Blair SN, Franklin BA, Myers J, Ross R (2017) Impact of cardiorespiratory fitness on all-cause and disease-specific mortality: advances since 2009. Prog Cardiovasc Dis 60:11-20. https://doi.org/10.1016/j.pcad.2017.03.001 
Haskell WL, Sims C, Myll J, Bortz WM, St Goar FG, Alderman EL (1993) Coronary artery size and dilating capacity in ultradistance runners. Circulation 87:1076-1082. https://doi.org/10.1161/ 01.cir.87.4.1076

He FJ, MacGregor GA (2011) Salt reduction lowers cardiovascular risk: meta-analysis of outcome trials. Lancet 378:380-382. https://doi.org/10.1016/s0140-6736(11)61174-4

He FJ, Li J, Macgregor GA (2013) Effect of longer term modest salt reduction on blood pressure: Cochrane systematic review and meta-analysis of randomised trials. BMJ (Clin Res Ed) 346: f1325. https://doi.org/10.1136/bmj.f1325

Hooper L, Martin N, Abdelhamid A, Davey Smith G (2015) Reduction in saturated fat intake for cardiovascular disease. Cochrane Database Syst Rev 6:Cd011737. https://doi.org/10.1002/ 14651858.Cd011737

$\mathrm{Hu}$ Y et al (2018) Smoking cessation, weight change, type 2 diabetes, and mortality. N Engl J Med 379:623-632. https://doi.org/10.1056/NEJMoa1803626

Hughes JR, Stead LF, Hartmann-Boyce J, Cahill K, Lancaster T (2014) Antidepressants for smoking cessation. Cochrane Database of Syst Rev 1:Cd000031. https://doi.org/10.1002/ 14651858.CD000031.pub4

Imamura F, Micha R, Wu JH, de Oliveira Otto MC, Otite FO, Abioye AI, Mozaffarian D (2016) Effects of saturated fat, polyunsaturated fat, monounsaturated fat, and carbohydrate on glucoseinsulin homeostasis: a systematic review and meta-analysis of randomised controlled feeding trials. PLoS Med 13:e1002087. https://doi.org/10.1371/journal.pmed.1002087

Jakobsen MU et al (2009) Major types of dietary fat and risk of coronary heart disease: a pooled analysis of 11 cohort studies. Am J Clin Nutr 89:1425-1432. https://doi.org/10.3945/ajen.2008. 27124

Katan MB, Grundy SM, Willett WC (1997) Should a low-fat, high-carbohydrate diet be recommended for everyone? Beyond low-fat diets. N Engl J Med 337:563-566; discussion 566-567

Katzmarzyk PT, Church TS, Blair SN (2004) Cardiorespiratory fitness attenuates the effects of the metabolic syndrome on all-cause and cardiovascular disease mortality in men. Arch Intern Med 164:1092-1097. https://doi.org/10.1001/archinte.164.10.1092

Khera AV et al (2016) Genetic risk, adherence to a healthy lifestyle, and coronary disease. N Engl J Med 375:2349-2358. https://doi.org/10.1056/NEJMoa1605086

Kodama S et al (2007) Effect of aerobic exercise training on serum levels of high-density lipoprotein cholesterol: a meta-analysis. Arch Intern Med 167:999-1008. https://doi.org/10.1001/ archinte.167.10.999

Lanter BB, Sauer K, Davies DG (2014) Bacteria present in carotid arterial plaques are found as biofilm deposits which may contribute to enhanced risk of plaque rupture. mBio 5:e01206e01214. https://doi.org/10.1128/mBio.01206-14

Latouche C, Jowett JB, Carey AL, Bertovic DA, Owen N, Dunstan DW, Kingwell BA (2013) Effects of breaking up prolonged sitting on skeletal muscle gene expression. J Appl Physiol (Bethesda, MD 1985) 114:453-460. https://doi.org/10.1152/japplphysiol.00978.2012

Lear SA et al (2017) The effect of physical activity on mortality and cardiovascular disease in 130,000 people from 17 high-income, middle-income, and low-income countries: the PURE study. Lancet 390:2643-2654. https://doi.org/10.1016/s0140-6736(17)31634-3

Lee DC et al (2011) Long-term effects of changes in cardiorespiratory fitness and body mass index on all-cause and cardiovascular disease mortality in men: the aerobics center longitudinal study. Circulation 124:2483-2490. https://doi.org/10.1161/circulationaha.111.038422

Li Y et al (2015) Saturated fats compared with unsaturated fats and sources of carbohydrates in relation to risk of coronary heart disease: a prospective cohort study. J Am Coll Cardiol 66:1538-1548. https://doi.org/10.1016/j.jacc.2015.07.055

Lloyd-Jones DM et al (2010) Defining and setting national goals for cardiovascular health promotion and disease reduction: the American Heart Association's strategic impact goal through 2020 and beyond. Circulation 121:586-613. https://doi.org/10.1161/circulationaha.109.192703 
Lollgen H, Bockenhoff A, Knapp G (2009) Physical activity and all-cause mortality: an updated meta-analysis with different intensity categories. Int J Sports Med 30:213-224. https://doi.org/ $10.1055 / \mathrm{s}-0028-1128150$

Lowe GD, Drummond MM, Forbes CD, Barbenel JC (1980) The effects of age and cigarettesmoking on blood and plasma viscosity in men. Scott Med J 25:13-17. https://doi.org/10.1177/ 003693308002500103

Lv X, Sun J, Bi Y, Xu M, Lu J, Zhao L, Xu Y (2015) Risk of all-cause mortality and cardiovascular disease associated with secondhand smoke exposure: a systematic review and meta-analysis. Int J Cardiol 199:106-115. https://doi.org/10.1016/j.ijcard.2015.07.011

Markuljak I, Ivankova J, Kubisz P (1995) Thrombomodulin and von Willebrand factor in smokers and during smoking. Nouv Rev Fr Hematol 37:137-139

Mensink RP, Zock PL, Kester AD, Katan MB (2003) Effects of dietary fatty acids and carbohydrates on the ratio of serum total to HDL cholesterol and on serum lipids and apolipoproteins: a meta-analysis of 60 controlled trials. Am J Clin Nutr 77:1146-1155. https://doi.org/10.1093/ajen/77.5.1146

Miller GJ, Bauer KA, Cooper JA, Rosenberg RD (1998) Activation of the coagulant pathway in cigarette smokers. Thromb Haemost 79:549-553

Miller PE, van Elswyk M, Alexander DD (2014) Long-chain omega-3 fatty acids eicosapentaenoic acid and docosahexaenoic acid and blood pressure: a meta-analysis of randomized controlled trials. Am J Hypertens 27:885-896. https://doi.org/10.1093/ajh/hpu024

Moholdt T, Lavie CJ, Nauman J (2017) Interaction of physical activity and body mass index on mortality in coronary heart disease: data from the Nord-Trondelag health study. Am J Med 130:949-957. https://doi.org/10.1016/j.amjmed.2017.01.043

Moholdt T, Lavie CJ, Nauman J (2018) Sustained physical activity, not weight loss, associated with improved survival in coronary heart disease. J Am Coll Cardiol 71:1094-1101. https://doi.org/ 10.1016/j.jacc.2018.01.011

Mons U et al (2015) Impact of smoking and smoking cessation on cardiovascular events and mortality among older adults: meta-analysis of individual participant data from prospective cohort studies of the CHANCES consortium. BMJ (Clin Res Ed) 350:h1551. https://doi.org/10. 1136/bmj.h1551

Moore SC et al (2012) Leisure time physical activity of moderate to vigorous intensity and mortality: a large pooled cohort analysis. PLoS Med 9:e1001335. https://doi.org/10.1371/ journal.pmed.1001335

Mozaffarian D, Katan MB, Ascherio A, Stampfer MJ, Willett WC (2006) Trans fatty acids and cardiovascular disease. N Engl J Med 354:1601-1613. https://doi.org/10.1056/NEJMra054035

Nauman J, Tauschek LC, Kaminsky LA, Nes BM, Wisloff U (2017) Global fitness levels: findings from a web-based surveillance report. Prog Cardiovasc Dis 60:78-88. https://doi.org/10.1016/j. pcad.2017.01.009

Nes BM, Gutvik CR, Lavie CJ, Nauman J, Wisloff U (2017) Personalized activity intelligence (PAI) for prevention of cardiovascular disease and promotion of physical activity. Am J Med 130:328-336. https://doi.org/10.1016/j.amjmed.2016.09.031

O'Donovan G, Stamatakis E, Stensel DJ, Hamer M (2018) The importance of vigorous-intensity leisure-time physical activity in reducing cardiovascular disease mortality risk in the obese. Mayo Clin Proc 93:1096-1103. https://doi.org/10.1016/j.mayocp.2018.01.016

Pandey A et al (2016) Continuous dose-response association between sedentary time and risk for cardiovascular disease: a meta-analysis. JAMA Cardiol 1:575-583. https://doi.org/10.1001/ jamacardio.2016.1567

Piepoli MF et al (2016) 2016 European guidelines on cardiovascular disease prevention in clinical practice: the sixth joint task force of the European Society of Cardiology and Other Societies on cardiovascular disease prevention in clinical practice (constituted by representatives of 10 societies and by invited experts)developed with the special contribution of the European Association for Cardiovascular Prevention \& Rehabilitation (EACPR). Eur Heart $\mathrm{J}$ 37:2315-2381. https://doi.org/10.1093/eurheartj/ehw106

Poggio R, Gutierrez L, Matta MG, Elorriaga N, Irazola V, Rubinstein A (2015) Daily sodium consumption and CVD mortality in the general population: systematic review and meta-analysis 
of prospective studies. Public Health Nutr 18:695-704. https://doi.org/10.1017/ s1368980014000949

Pulsford RM, Blackwell J, Hillsdon M, Kos K (2017) Intermittent walking, but not standing, improves postprandial insulin and glucose relative to sustained sitting: a randomised cross-over study in inactive middle-aged men. J Sci Med Sport 20:278-283. https://doi.org/10.1016/j. jsams.2016.08.012

Rahman MM, Laher I (2007) Structural and functional alteration of blood vessels caused by cigarette smoking: an overview of molecular mechanisms. Curr Vasc Pharmacol 5:276-292

Rasmussen BM et al (2006) Effects of dietary saturated, monounsaturated, and n-3 fatty acids on blood pressure in healthy subjects. Am J Clin Nutr 83:221-226. https://doi.org/10.1093/ajcn/83. 2.221

Raveendran M et al (2004) Cigarette suppresses the expression of $\mathrm{P} 4 \mathrm{H} \alpha$ and vascular collagen production. Biochem Biophys Res Commun 323:592-598. https://doi.org/10.1016/j.bbrc.2004. 08.129

Reiner Z (2013) Statins in the primary prevention of cardiovascular disease. Nat Rev Cardiol 10:453-464. https://doi.org/10.1038/nrcardio.2013.80

Reiner Z et al (2011) ESC/EAS guidelines for the management of dyslipidaemias: the task force for the management of dyslipidaemias of the European Society of Cardiology (ESC) and the European Atherosclerosis Society (EAS). Eur Heart J 32:1769-1818. https://doi.org/10.1093/ eurheartj/ehr158

Reiner Z, Laufs U, Cosentino F, Landmesser U (2019) The year in cardiology 2018: prevention. Eur Heart J 40:336-344. https://doi.org/10.1093/eurheartj/ehy894

Reynolds A, Mann J, Cummings J, Winter N, Mete E, Te Morenga L (2019) Carbohydrate quality and human health: a series of systematic reviews and meta-analyses. Lancet 393:434-445. https://doi.org/10.1016/s0140-6736(18)31809-9

Riccardi G, Costabile G (2019) Carbohydrate quality is key for a healthy and sustainable diet. Nat Rev Endocrinol 15:257-258. https://doi.org/10.1038/s41574-019-0190-x

Riccardi G, Rivellese AA (1991) Effects of dietary fiber and carbohydrate on glucose and lipoprotein metabolism in diabetic patients. Diabetes Care 14:1115-1125. https://doi.org/10.2337/ diacare.14.12.1115

Riccardi G, Rivellese AA, Giacco R (2008) Role of glycemic index and glycemic load in the healthy state, in prediabetes, and in diabetes. Am J Clin Nutr 87:269s-274s. https://doi.org/10. 1093/ajcn/87.1.269S

Rivellese AA, Giacco R, Costabile G (2012) Dietary carbohydrates for diabetics. Curr Atheroscler Rep 14:563-569. https://doi.org/10.1007/s11883-012-0278-4

Sacks FM, Katan M (2002) Randomized clinical trials on the effects of dietary fat and carbohydrate on plasma lipoproteins and cardiovascular disease. Am J Med 113(Suppl 9B):13s-24s. https:// doi.org/10.1016/s0002-9343(01)00987-1

Sacks FM et al (2017) Dietary fats and cardiovascular disease: a presidential advisory from the American Heart Association. Circulation 136:e1-e23. https://doi.org/10.1161/cir. 0000000000000510

Sahlen A et al (2019) Air pollution in relation to very short-term risk of ST-segment elevation myocardial infarction: case-crossover analysis of SWEDEHEART. Int J Cardiol 275:26-30. https://doi.org/10.1016/j.ijcard.2018.10.069

Sattelmair J, Pertman J, Ding EL, Kohl HW 3rd, Haskell W, Lee IM (2011) Dose response between physical activity and risk of coronary heart disease: a meta-analysis. Circulation 124:789-795. https://doi.org/10.1161/circulationaha.110.010710

Schwingshackl L, Strasser B, Hoffmann G (2011) Effects of monounsaturated fatty acids on cardiovascular risk factors: a systematic review and meta-analysis. Ann Nutr Metab 59:176-186. https://doi.org/10.1159/000334071

Seidelmann SB et al (2018) Dietary carbohydrate intake and mortality: a prospective cohort study and meta-analysis. Lancet Public Health 3:e419-e428. https://doi.org/10.1016/s2468-2667(18) 30135-x 
Siervo M, Lara J, Chowdhury S, Ashor A, Oggioni C, Mathers JC (2015) Effects of the dietary approach to stop hypertension (DASH) diet on cardiovascular risk factors: a systematic review and meta-analysis. Br J Nutr 113:1-15. https://doi.org/10.1017/s0007114514003341

Siri-Tarino PW, Sun Q, Hu FB, Krauss RM (2010) Meta-analysis of prospective cohort studies evaluating the association of saturated fat with cardiovascular disease. Am J Clin Nutr 91:535-546. https://doi.org/10.3945/ajcn.2009.27725

Skogstad M, Johannessen HA, Tynes T, Mehlum IS, Nordby KC, Lie A (2016) Systematic review of the cardiovascular effects of occupational noise. Occup Med 66:10-16. https://doi.org/10. 1093/occmed/kqv148

Smith CJ, Fischer TH (2001) Particulate and vapor phase constituents of cigarette mainstream smoke and risk of myocardial infarction. Atherosclerosis 158:257-267

Sofi F, Macchi C, Abbate R, Gensini GF, Casini A (2014) Mediterranean diet and health status: an updated meta-analysis and a proposal for a literature-based adherence score. Public Health Nutr 17:2769-2782. https://doi.org/10.1017/s1368980013003169

Stamler J et al (2018) Relation of dietary sodium (salt) to blood pressure and its possible modulation by other dietary factors: the INTERMAP study. Hypertension 71:631-637. https://doi.org/10. 1161/hypertensionaha.117.09928

Stanhope KL et al (2009) Consuming fructose-sweetened, not glucose-sweetened, beverages increases visceral adiposity and lipids and decreases insulin sensitivity in overweight/obese humans. J Clin Invest 119:1322-1334. https://doi.org/10.1172/jci37385

Stead LF, Perera R, Bullen C, Mant D, Hartmann-Boyce J, Cahill K, Lancaster T (2012) Nicotine replacement therapy for smoking cessation. Cochrane Database Syst Rev 11:Cd000146. https:// doi.org/10.1002/14651858.CD000146.pub4

Summers LK et al (2002) Substituting dietary saturated fat with polyunsaturated fat changes abdominal fat distribution and improves insulin sensitivity. Diabetologia 45:369-377. https:// doi.org/10.1007/s00125-001-0768-3

Teixeira LR et al (2019) WHO/ILO work-related burden of disease and injury: protocol for systematic reviews of exposure to occupational noise and of the effect of exposure to occupational noise on cardiovascular disease. Environ Int 125:567-578. https://doi.org/10.1016/j. envint.2018.09.040

Teo KK et al (2006) Tobacco use and risk of myocardial infarction in 52 countries in the INTERHEART study: a case-control study. Lancet 368:647-658. https://doi.org/10.1016/ s0140-6736(06)69249-0

Thijssen DH, Cable NT, Green DJ (2012) Impact of exercise training on arterial wall thickness in humans. Clin Sci 122:311-322. https://doi.org/10.1042/cs20110469

Thomas DE, Elliott EJ (2010) The use of low-glycaemic index diets in diabetes control. Br J Nutr 104:797-802. https://doi.org/10.1017/s0007114510001534

US Department of Agriculture (2016) Agricultural research service, national nutrient database for standard reference. https://ndb.nal.usda.gov/ndb/search/list

van Horn L et al (2016) Recommended dietary pattern to achieve adherence to the American Heart Association/American College of Cardiology (AHA/ACC) guidelines: a scientific statement from the American Heart Association. Circulation 134:e505-e529. https://doi.org/10.1161/cir. 0000000000000462

Vessby B et al (2001) Substituting dietary saturated for monounsaturated fat impairs insulin sensitivity in healthy men and women: the KANWU study. Diabetologia 44:312-319

Vodak PA, Wood PD, Haskell WL, Williams PT (1980) HDL-cholesterol and other plasma lipid and lipoprotein concentrations in middle-aged male and female tennis players. Metab Clin Exp 29:745-752

Wang XL, Sim AS, Badenhop RF, McCredie RM, Wilcken DE (1996) A smoking-dependent risk of coronary artery disease associated with a polymorphism of the endothelial nitric oxide synthase gene. Nat Med 2:41-45 
Werner CM et al (2019) Differential effects of endurance, interval, and resistance training on telomerase activity and telomere length in a randomized, controlled study. Eur Heart J 40:34-46. https://doi.org/10.1093/eurheartj/ehy585

WHO (2013) Global action plan for the prevention and control of NCDs 2013-2020. WHO, Geneva

WHO Guidelines Approved by the Guidelines Review Committee (2012) Guideline: sodium intake for adults and children. World Health Organization, Geneva

Wood PD, Haskell W, Klein H, Lewis S, Stern MP, Farquhar JW (1976) The distribution of plasma lipoproteins in middle-aged male runners. Metab Clin Exp 25:1249-1257

$\mathrm{Xi} B$ et al (2015) Sugar-sweetened beverages and risk of hypertension and CVD: a dose-response meta-analysis. Br J Nutr 113:709-717. https://doi.org/10.1017/s0007114514004383

Zhong VW et al (2019) Associations of dietary cholesterol or egg consumption with incident cardiovascular disease and mortality. JAMA 321:1081-1095. https://doi.org/10.1001/jama. 2019.1572

Zhou L et al (2019) Salt intake and prevalence of overweight/obesity in Japan, China, the United Kingdom, and the United States: the INTERMAP study. Am J Clin Nutr 110:34. https://doi.org/ 10.1093/ajcn/nqz067

Zhu BQ, Parmley WW (1995) Hemodynamic and vascular effects of active and passive smoking. Am Heart J 130:1270-1275. https://doi.org/10.1016/0002-8703(95)90154-x

Zock PL, Blom WA, Nettleton JA, Hornstra G (2016) Progressing insights into the role of dietary fats in the prevention of cardiovascular disease. Curr Cardiol Rep 18:111. https://doi.org/10. 1007/s11886-016-0793-y

Open Access This chapter is licensed under the terms of the Creative Commons Attribution 4.0 International License (http://creativecommons.org/licenses/by/4.0/), which permits use, sharing, adaptation, distribution and reproduction in any medium or format, as long as you give appropriate credit to the original author(s) and the source, provide a link to the Creative Commons licence and indicate if changes were made.

The images or other third party material in this chapter are included in the chapter's Creative Commons licence, unless indicated otherwise in a credit line to the material. If material is not included in the chapter's Creative Commons licence and your intended use is not permitted by statutory regulation or exceeds the permitted use, you will need to obtain permission directly from the copyright holder.

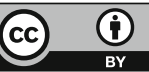

